Supporting Information for

\title{
Phosphorization Treatment Improves the Catalytic Activity and Durability of Platinum Catalysts toward Oxygen Reduction Reaction
}

\author{
Ruiyun Guo, Wei Bi, Ke Zhang, Yaming Liu, Chaoqi Wang, Yangzi Zheng, and \\ Mingshang Jin* \\ Frontier Institute of Science and Technology and State Key Laboratory of Multiphase Flow in Power \\ Engineering, Xi'an Jiaotong University, Xi'an, Shaanxi 710049, China. E-mail: jinm@mail.xjtu.edu.cn
}



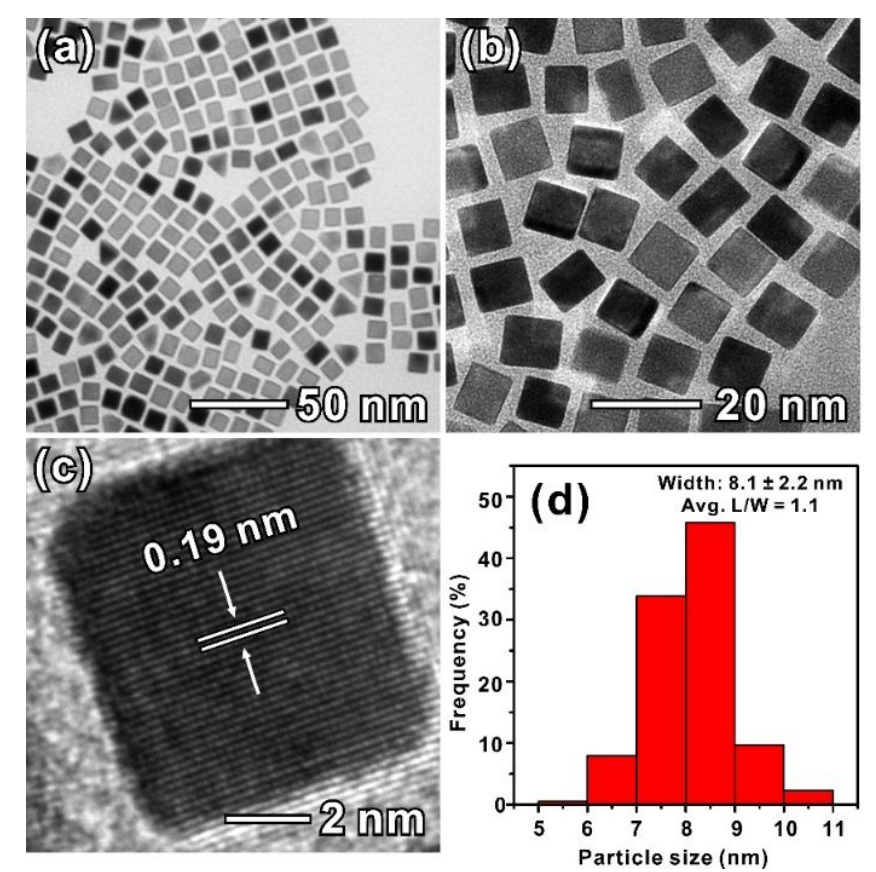

Figure S1. (a, b) TEM and (c) HRTEM images of as-prepared Pt nanocubes. (d) The corresponding histogram of size distribution was counted from 500 particles. 

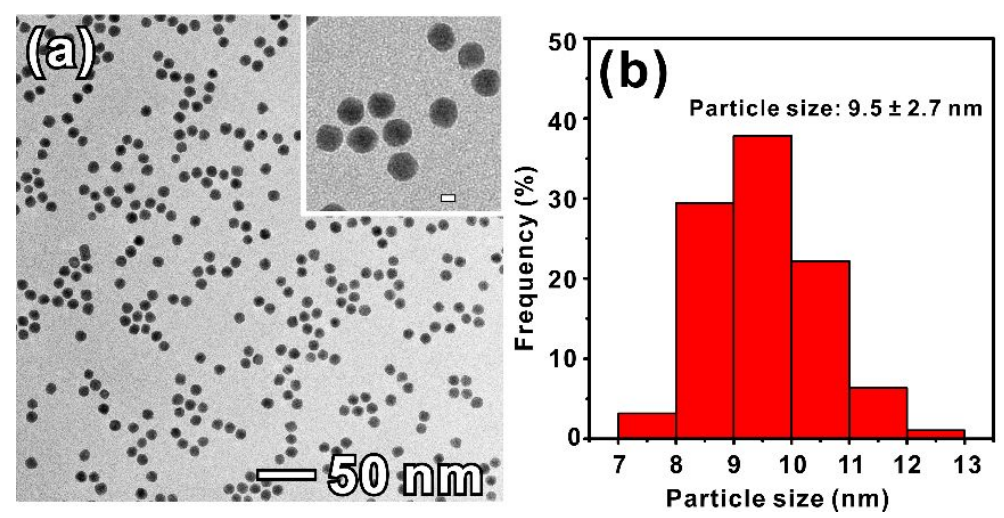

Figure S2. (a) Low magnification TEM image of the obtained $\mathrm{Pt}_{2} \mathrm{P}$ nanocrystals. Scale bar in inset, $5 \mathrm{~nm}$. (b) The corresponding histogram of size distribution was counted from 500 particles. 


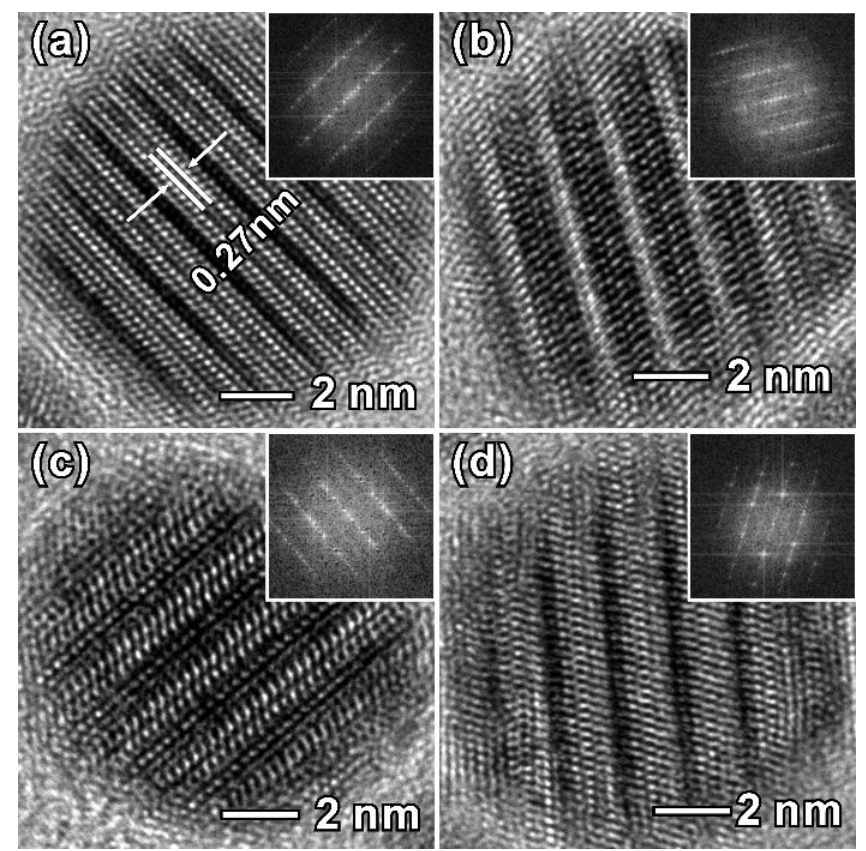

Figure S3. Additional HRTEM images and FFT patterns (insets) of $\mathrm{Pt}_{2} \mathrm{P}$ nanocrystals which containing abundant stacking faults. 


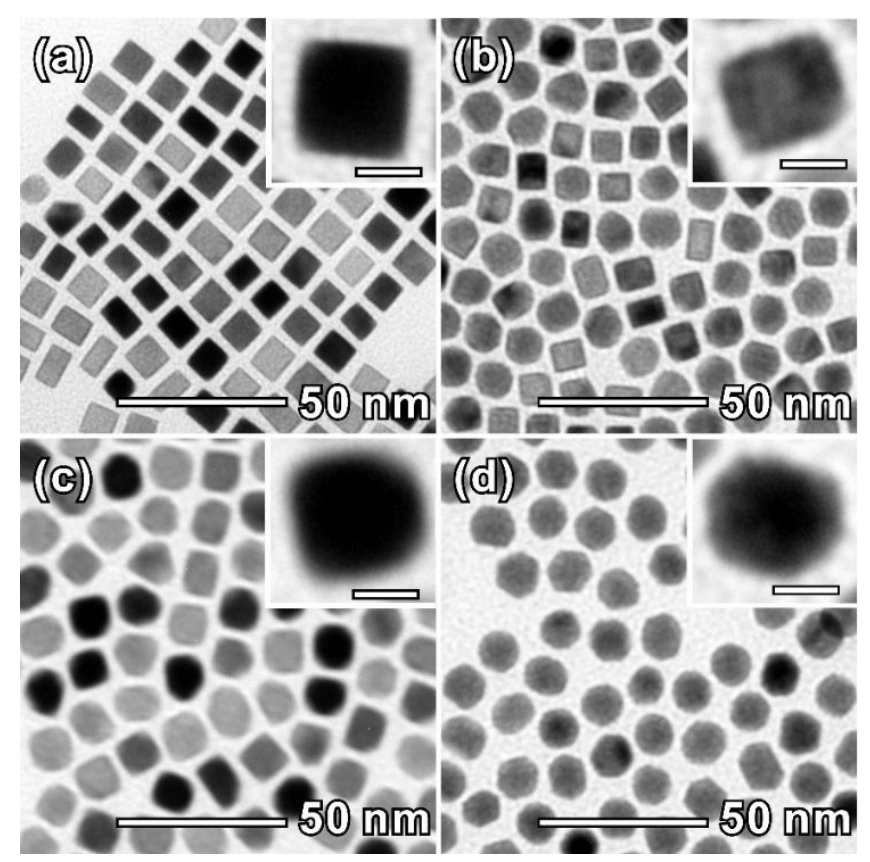

Figure S4. Time tracking TEM images during the synthesis of $\mathrm{Pt}_{2} \mathrm{P}$ nanocrystals with reaction time of (a) $1 \mathrm{~h}$, (b) $1.5 \mathrm{~h}$, (c) $2 \mathrm{~h}$ and (d) $2.5 \mathrm{~h}$. Insets show individual nanocrystal at higher magnification. The scale bars in the insets are $5 \mathrm{~nm}$. 


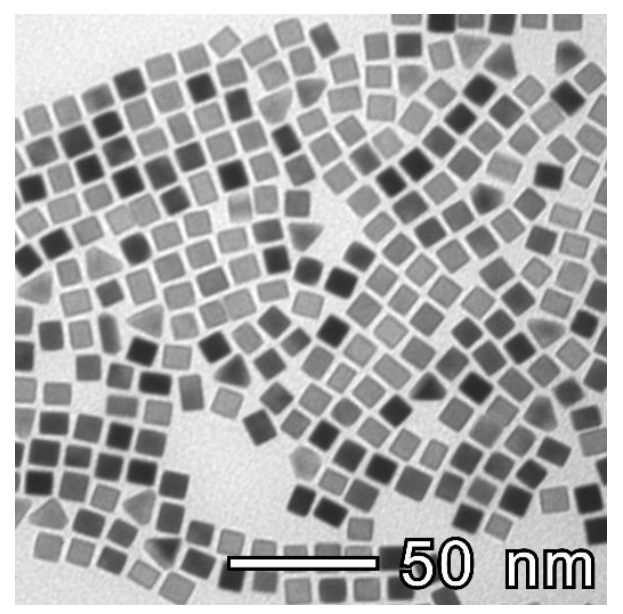

Figure S5. TEM image of the product obtained from a typical synthesis in the absence of TOP. 

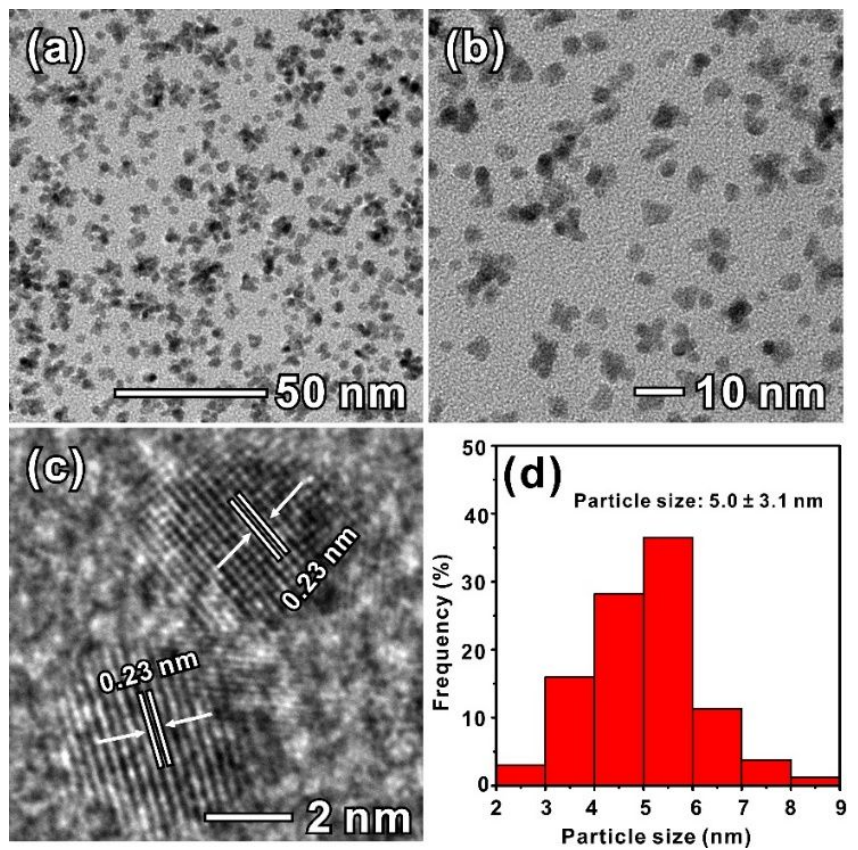

Figure S6. (a, b) TEM and (c) HRTEM images of as-prepared Pt nanoparticles. (d) The corresponding histogram of size distribution was counted from 500 nanoparticles. 

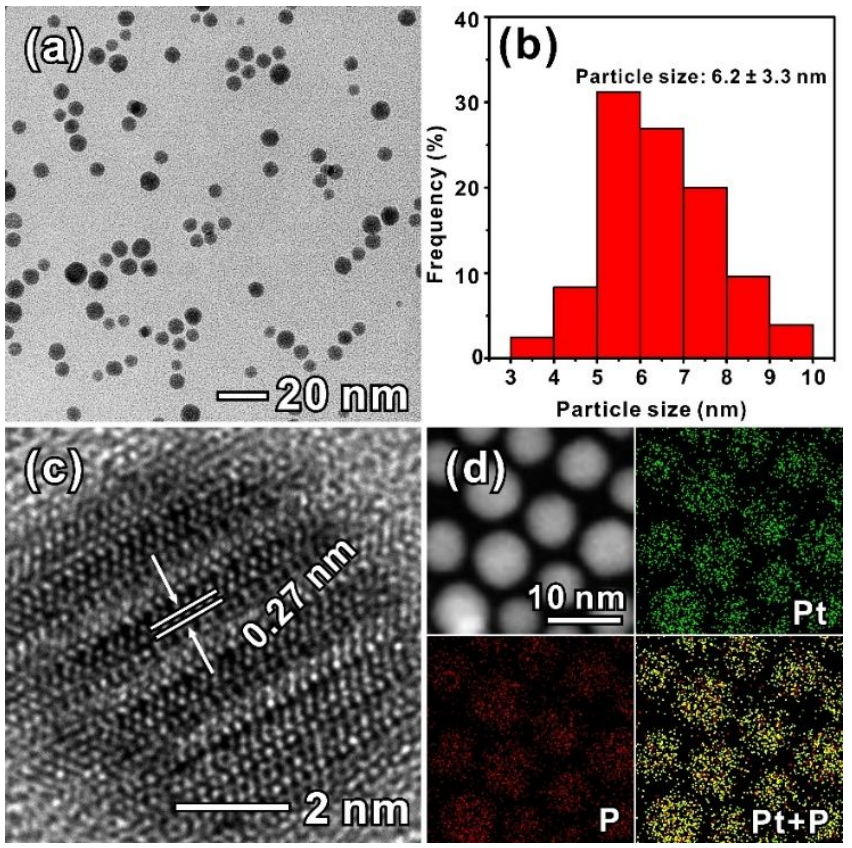

Figure S7. (a) TEM and (b) corresponding histogram of size distribution of $\mathrm{Pt}_{2} \mathrm{P}$ nanocrystals. (c) HRTEM image of an individual $\mathrm{Pt}_{2} \mathrm{P}$ nanocrystal. (d) EDS elemental mappings of $\mathrm{Pt}_{2} \mathrm{P}$ nanocrystals. 


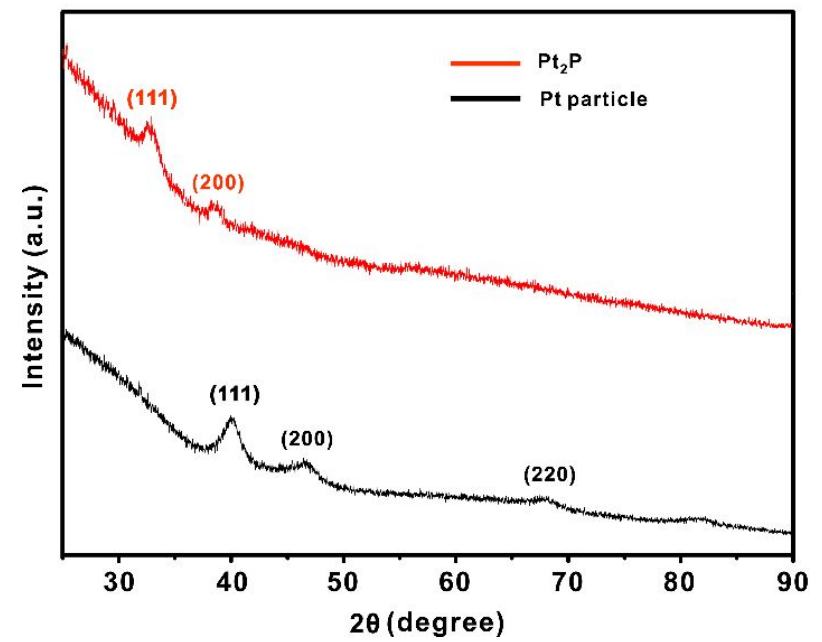

Figure S8. XRD patterns of obtained $\mathrm{Pt}$ nanoparticles (black line) and $\mathrm{Pt}_{2} \mathrm{P}$ nanocrystals (red line). 


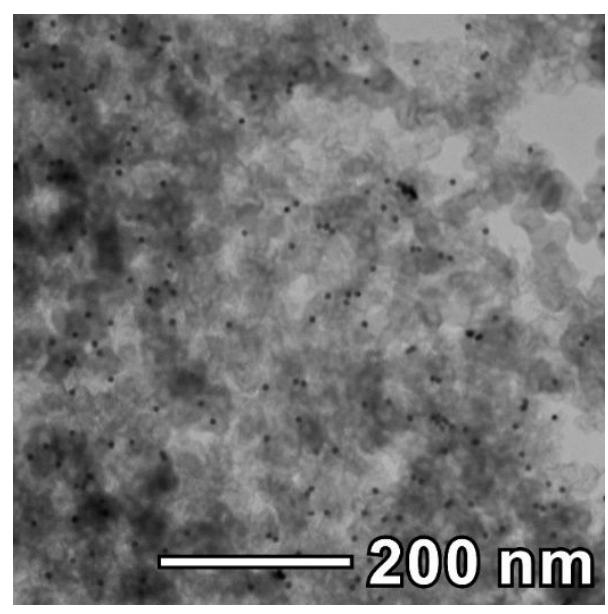

Figure S9. Low magnification TEM image of $6.2 \mathrm{~nm}-\mathrm{Pt}_{2} \mathrm{P}$ nanocrystal catalyst after ADTs of 30,000 cycles. 


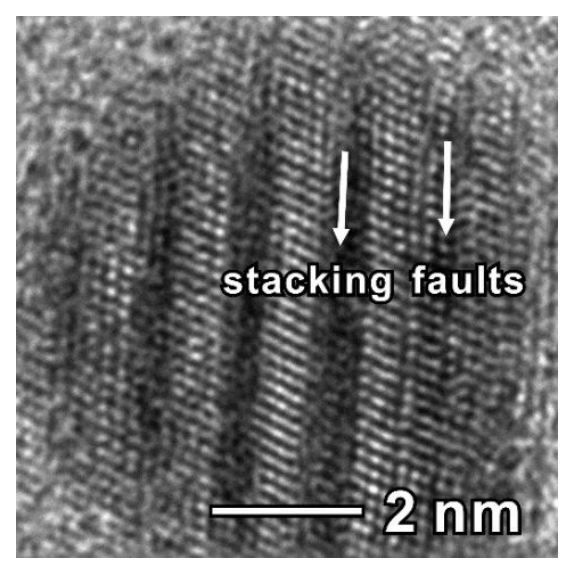

Figure S10. HRTEM image of $6.2 \mathrm{~nm}-\mathrm{Pt}_{2} \mathrm{P}$ nanocrystal catalyst after ADTs of 30,000 cycles. 


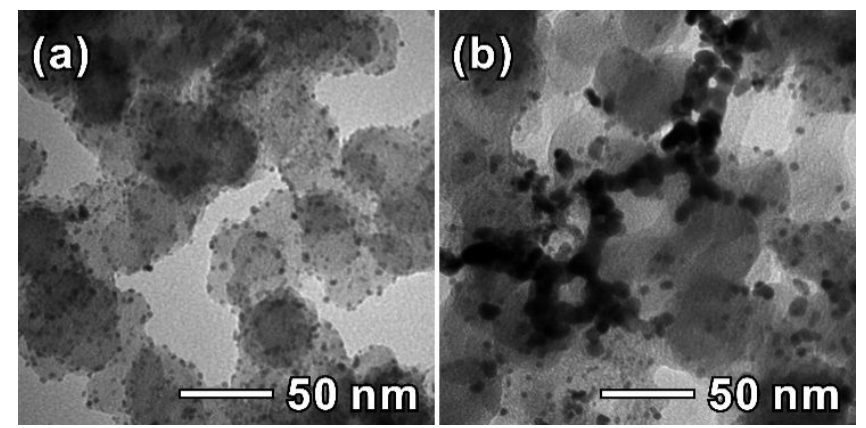

Figure S11. TEM images of the (a) pristine $\mathrm{Pt} / \mathrm{C}$ catalyst and (b) $\mathrm{Pt} / \mathrm{C}$ catalyst after ADTs of 30,000 cycles. 


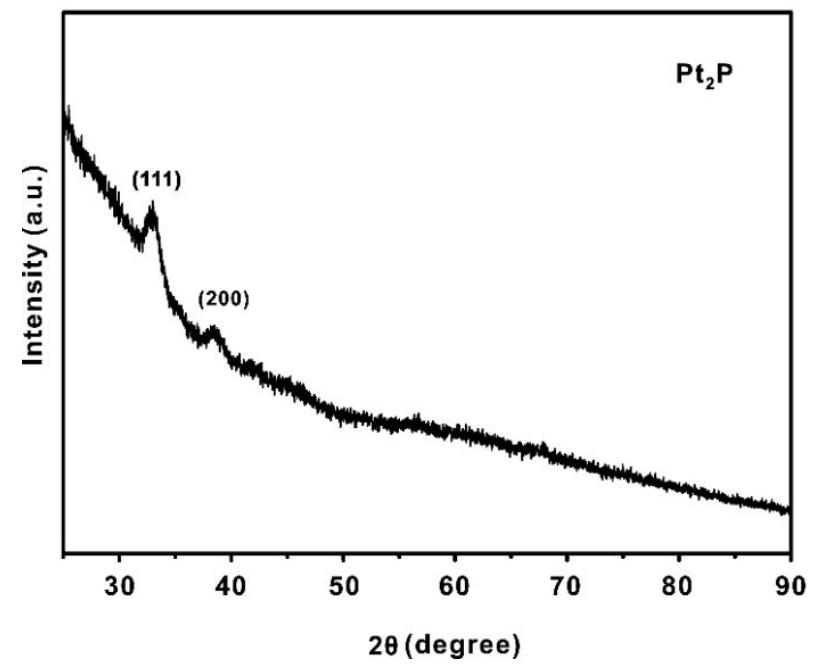

Figure S12. XRD pattern of $\mathrm{Pt}_{2} \mathrm{P}$ nanocrystal catalyst after ADTs of 30,000 cycles. 
Table S1. Calculated XRD parameters of $\mathrm{Pt}_{2} \mathrm{P}$ nanocrystals.

\begin{tabular}{cccc}
\hline $\begin{array}{c}2 \text { Theta } \\
\left({ }^{\circ}\right)\end{array}$ & $\begin{array}{c}\text { d-spacing } \\
(\AA)\end{array}$ & $\begin{array}{c}\text { Intensity } \\
(\text { a. u. })\end{array}$ & $(\mathrm{hkl})$ \\
\hline 32.818 & 2.7268 & 6525345.45 & 111 \\
38.075 & 2.3615 & 3299137.35 & 200 \\
54.943 & 1.6698 & 2325089.18 & 220 \\
65.511 & 1.4240 & 2771611.79 & 311 \\
68.802 & 1.3634 & 802012.80 & 222 \\
81.443 & 1.1808 & 387946.12 & 400 \\
\hline
\end{tabular}


Table S2. The atom content in $\mathrm{Pt}_{2} \mathrm{P}$ nanocrystals analyzed by ICP-MS.

\begin{tabular}{ccc}
\hline Element & Concentration $\left(10^{-6} \mathrm{~g} / \mathrm{ml}\right)$ & Uncertainly \\
\hline $\mathrm{Pt}$ & 2.318 & 0.06 \\
$\mathrm{P}$ & 0.181 & 0.021 \\
\hline
\end{tabular}


Table S3. ICP-MS analyses of the samples obtained at different reaction times.

\begin{tabular}{cccc}
\hline $\begin{array}{c}\text { Reaction time } \\
(\mathrm{h})\end{array}$ & $\begin{array}{c}\text { Pt concentration } \\
\left(10^{-6} \mathrm{~g} / \mathrm{ml}\right)\end{array}$ & $\begin{array}{c}\text { P concentration } \\
\left(10^{-6} \mathrm{~g} / \mathrm{ml}\right)\end{array}$ & $\begin{array}{c}\text { Approximate } \\
\text { composition }\end{array}$ \\
\hline 1.0 & 28.396 & 0.188 & $\mathrm{Pt}: \mathrm{P}=96: 4$ \\
1.5 & 21.791 & 0.385 & $\mathrm{Pt}: \mathrm{P}=90: 10$ \\
2.0 & 19.862 & 0.647 & $\mathrm{Pt}: \mathrm{P}=83: 17$ \\
2.5 & 29.378 & 1.317 & $\mathrm{Pt}: \mathrm{P}=78: 22$ \\
\hline
\end{tabular}


Table S4. Specific ECSAs and $E_{1 / 2}$ values of different catalysts.

\begin{tabular}{ccc}
\hline Samples & Specific ECSA $\left(\mathrm{m}^{2} / \mathrm{g}\right)$ & $\mathrm{E}_{1 / 2}(\mathrm{~V})$ \\
\hline Pt cube & 18.6 & 0.839 \\
Pt particle & 48.5 & 0.853 \\
$\mathrm{Pt} / \mathrm{C}$ & 68.2 & 0.877 \\
$\mathrm{Pt}_{2} \mathrm{P}-9.5 \mathrm{~nm}$ & 41.6 & 0.905 \\
$\mathrm{Pt}_{2} \mathrm{P}-6.2 \mathrm{~nm}$ & 52 & 0.927 \\
\hline
\end{tabular}

